\title{
The Challenges and Limitations of Chemical Analysis of Particulate Pigments of Very Low Solubility
}

\author{
Ole Olsen
}

Medico Chemical Lab ApS, Vedbaek, Denmark

\begin{abstract}
When performing a chemical analysis of colorants in tattoo products, specific degradation products as well as impurity patterns can be predicted. Mislabeling or false declarations can also be avoided using this test. It is notable that pigment identification in tattoo products may serve as a precursory technique to recognize the colorants contained in a patient's tattoo prior to laser removal therapy. In contrast to the analysis of banned pigments, positive identification of pigments will normally require few reference substances. Given the fact that tattoo pigments are nearly insoluble in water and many organic solvents, different chemical pigment analyses are outlined and evaluated. Related publications from the study of art are also mentioned. It is recommended that access to comprehensive pigment standards and spectroscopic databanks should be offered to laboratories dealing with tattoo product analysis in the future.
\end{abstract}

(C) 2015 S. Karger AG, Basel

\section{Introduction}

Tattoo products consist of colorants dispersed in a suitable matrix, which is normally a solution of water, glycerin, alcohol, resins, surfactants, and witch hazel. Different types of colorants are responsible for the final motif of the tattoo. The concentrations of the colorants in tattoo products are often above $50 \%(\mathrm{w} / \mathrm{w})$ [1]. As tattoos are meant to last for a lifetime, the colorants have to be very stable after placement in the dermis. This means that the colorants have to be insoluble in water. If not, they will be washed away during contact with the tissue. When a colorant is insoluble in its own matrix, it is called a pigment. Today, approximately $80 \%$ of the pigments in tattoo products are synthetic organic molecules, mostly azo pigments and polycyclic compounds [2]. The most frequently used inorganic pigments are titanium dioxide, barium sulfate, and iron oxides [3]. 
Organic pigments are favored for tattooing because of their high tinting strengths, light fastness, enzymatic resistance, dispersion, and relatively inexpensive production [4]. In order to secure the safety and quality of tattoo products for the consumer, different regulatory guidelines have been developed during the last 20 years. The European guideline from Council of Europe, 2008, Resolution ResAP(2008)2 on tattoos and permanent make-up [5] is the most significant example to be mentioned. According to this guideline, the only identification required to verify the pigments in tattoo products is the color index (CI) number printed on the label. No chemical identification of the pigment is needed. As some pigments (mostly azo pigments) are known to degrade into potentially harmful substances, such as primary amines, the guideline contains a list of 35 banned pigments suspected of being potential precursors of degradation products, such as the carcinogenic primary amines o-anisidine, 5-nitro-o-toluidine, 4-chloro-o-toluidine, and 3,3-dichlorobenzidine [1]. To fulfill this requirement, a reference sample of all 35 different pigments is needed for the chemical analysis of pigments. Engel et al. [6] and Vasold et al. [7] conducted in vitro experiments to show that potentially toxic/carcinogenic decomposition products form when using a typical Q-switched Nd:YAG $(532 \mathrm{~nm})$ laser on two common red tattoo inks.

As the market for tattoo products is huge and nontransparent, it could be desirable for positive identification of the pigments in tattoo products to be established as a standard safety test. This would also avoid mislabeling or false declarations. In contrast to the analysis of the banned pigments, positive identification of pigments will normally require few reference pigments, depending of the type of tattoo product.

Another important reason to know the identity of the pigments in tattoo products is related to tattoo removal. Here, it is necessary to know the absorption wavelengths of the actual pig- ments so that the laser light can be matched to the absorption spectra of the tattooed compounds. In other words, the identification of pigments in tattoo products might serve as an important precursory technique prior to laser removal therapy [4]. As some impurities are related to specific pigments [8], positive pigment identification could predict the impurity pattern of the actual tattoo product. As noted by Bäumler et al. [9], the analysis of pigments using standard methods has turned out to be very troublesome because of their extremely low solubility. This is a general problem in all analytical quantification methods in which sample preparations are included. If the solubility is less than $1 \mathrm{mg} / \mathrm{ml}$ at $22^{\circ} \mathrm{C}$, the compound is generally characterized as insoluble [1]. It has been observed that pigments are generally considered as insoluble in water and also partially insoluble in most organic solvents. In many of the chemical methods described below, the pigment concentration is very low and close to the insoluble limit.

When setting up an analytical standard method for the analysis of pigments in tattoo products, one also has to take into account the variable solubility of the pigments. In other words, the pigments with the lowest solubility will be responsible for the final setting of the pigment concentration in the analytical standard. If quantification of the pigment is needed, one has to ensure that all pigments are totally dissolved when making the calibration curve.

As reference pigments are necessary for the performance of analytical procedures, it should be noted that access to a suitable selection of reference pigments seems to be a serious problem for routine laboratories.

\section{Chemical Analyses of Tattoo Pigments}

During the last 20 years, several investigations of the pigment content of tattoo products have been performed, and a survey of the many pigments 
used in tattoo inks is given in $[1,10,11]$. Inorganic pigments can be analyzed relatively straightforwardly with techniques such as energy-dispersive spectrometry, X-ray fluorescence, or X-ray diffraction $[4,12]$. In the following sections, different published analytical methods are listed and discussed. The analytical methods are arranged according to their analyzing principle.

\section{Fourier Transform Infrared Spectroscopy}

Poon et al. [4] carried out an analytical procedure in which organic pigments were identified in situ and post-tattoo procedure by Fourier transform infrared spectroscopy (FTIR). The pigments were filtrated from the tattoo ink, finely ground with analytical-grade $\mathrm{KBr}$, and pressed into pellets. Peak wave numbers and spectra were visually compared and cross-referenced against the active reference material and existing databases. The method was used as a complementary technique to Raman spectroscopy to confirm the identity of the pigments.

Bäumler et al. [9] analyzed 41 tattoo inks and identified 16 different pigments using FTIR. The pigments were placed in a $\mathrm{KBr}$ disc (pigment concentration $0.1 \% \mathrm{w} / \mathrm{w}$ ) and identified by computer-assisted pattern comparison with original pigment spectra from databanks. According to the authors, the analysis of the pigments by using standard methods turned out to be very troublesome because of the pigments' extremely low solubility or volatility. Using this method, 16 different pigments were determined.

Vila and Garcia [13] investigated the composition of red pigments and inks for the characterization and differentiation of prints. They used a combination of FTIR and an energy-dispersive $\mathrm{X}$-ray-analyzing system (scanning electron microscopy-energy-dispersive X-ray (SEM-EDX)), which are common techniques available in museums and at centers related to the study of artwork. FTIR provides information about the organic and some inorganic components of pigments and inks, whereas SEM-EDX provides the elemental composition of the material. The mid-IR range is very useful for detecting the fingerprint of organic materials, although it does not allow the identification of inorganic pigments with absorptions below $400 \mathrm{~cm}^{-1}$, such as vermilion, titanium, and cadmium pigments. In the study, the inks were spread on top of a pure $\mathrm{KBr}$ pellet. The authors concluded that due to the complexity of the synthetic organic pigments, a combination of the two methods (FTIR and SEM-EDX) was needed for the identification of all components in the actual inks.

Schaening et al. [14], from the Academy of Fine Arts, used FTIR for the identification and classification of synthetic organic pigments in a collection from the 19th and 20th centuries. Different organic pigments could be detected by FTIR in combination with a diamond sample cell. The identification was based on reference material taken from a large pigment database. In another study [15], the authors used a multivariate data analysis of FTIR spectra to support the classification of synthetic organic pigments. FTIR seems to be a very useful method for the examination of pigments in old paintings. Learner [16], from the Tate Gallery, used FTIR to analyze pigments in paintings, and FTIR has proven to be a very useful method for rapid differentiation between the main types of 20th-century paint media. In forensic paint examinations, FTIR analysis seems to be the routine choice. The FBI Laboratory for Paints and Polymers [17] has developed a standard operating procedure regarding the identification of pigments in paints. The paint is placed between two inactive IR windows or in a diamond cell.

\section{High-Pressure Liquid Chromatography}

Using high-pressure liquid chromatography (HPLC) and diode array detection (DAD), Bhakti and Petigara [18] identified the two major classes of organic pigments, along with benzimidazolone-based organic pigments for orange and brown ink colors. The retention times and spectral data for the pigments in the examined tattoo inks were correlated with those of reference 
standards. The complexity of the ink mixtures did complicate the correlation with the specific pigments.

Investigating the photodecomposition of Pigment Yellow 74 (PY74), Cui et al. [19] analyzed seven yellow tattoo inks. The inks were mixed with water and extracted with methylene chloride. The samples were analyzed using HPLC equipped with a diode array detector. PY74 was identified in six of the seven inks by comparing the HPLC retention times and absorbance spectra of the eluting components with those of authentic PY74.

Engel et al. [20] have established a protocol to analyze tattoo pigments in situ by HPLC and DAD. Using a rather complicated extraction method, nearly complete recovery from aqueous suspension was achieved. This is an interesting study because quantification of the pigments was included.

Vasold et al. [7] used the same instrumentation when looking at degradation products after laser light cleavage of the two pigments CI 12315 and CI 12460. The pigments were identified by their retention times and spectral data in comparison with those of reference pigments.

A well-known method to identify and quantify organic pigments in textiles is described in Textilien-Nachweis von Dispersionsfarbstoffen [21]. Some laboratories are using this method for the identification of the 35 banned pigments, as stated in the Council of Europe, 2008, Resolution ResAP(2008) 2 on tattoos and permanent makeup. The method uses HPLC and DAD, with methanol as the extraction solvent. The retention times and spectral data for the pigments in the examined tattoo inks are correlated with those of reference standards. The detection limit is approximately 1-2 mg/l. As the method was developed for the analysis of textiles, modifications have to be implemented for the analysis of tattoo inks.

\section{Raman Spectroscopy}

Poon et al. [4] carried out an analytical procedure in which organic pigments were identified in situ and post-tattoo procedure by Raman spectroscopy. During the sample preparation, separation from the carrier solution was necessary, as overwhelming fluorescence was observed when the tattoo inks were simply smeared onto glass slides and analyzed by Raman spectroscopy. Using this method, $\mathrm{TiO}_{2}$ was identified separately. Raman analysis was performed on reference material and tattoo pigments separated from the pre-mixed ink by focusing the laser on the sample mounted onto the glass slides. Beyond the rather comprehensive sample preparation, the method seems suitable for routine purposes. Vandenabeele et al. [12] investigated the pigments in different paintings using a fiber-optic probe combined with a Raman spectrophotometer. In this direct analysis, the probe was placed approximately $1 \mathrm{~cm}$ above the object (which could be tattoo ink evaporated on a glass plate). Several pigments in the painting could be identified using reference samples. The method seems interesting, as it is a direct and fast analysis.

\section{Different Time-of-Flight-Based Analytical Methods}

Hauri [8] analyzed 167 tattoo ink samples for their content of pigments; aromatic amines; carcinogenic, mutagenic, and reprotoxic substances; and preservatives. The method used for qualitative analysis of organic pigments was matrixassisted laser desorption ionization time-of-flight (TOF) mass spectrometry (MS). This allowed most of the pigments that were used to be analyzed directly from the dyes themselves, without adding a matrix. Along with the poor solubility, the biggest problem in the analysis lay in obtaining reference substances.

The Mass Spectrometry Application Group at JEOL Ltd. [22] has described a method in which Pigment Yellow 83 and Pigment Red 144 are identified using a direct exposure probe (DEP) and a TOF gas chromatography mass spectrometer (AccuTOF GCv 4G). Regarding 
the DEP, the analyte solution (or suspension) is placed on a coiled platinium filament at the end of the probe. The probe is introduced into the ion source, and the analyte is rapidly heated and vaporized by passing an electric current through the filament. Since the analyte is vaporized by rapid heating with the DEP, this method is efficient and suitable for the analysis of high-boiling-point and thermally labile compounds. As the method has only been tested on two different pigments, it is unknown whether it is suitable for analyses of pigment mixtures.

Oxidative degradation of the azo pigment CI 13020 was studied by Djelal et al. [23] using direct analysis in real time TOF MS. The method is similar to the method described above, and again, only data based on experiments on one pigment are available. Fang et al. [24] have developed a highly advanced method for the lowlevel detection of organic pigments. The method is based on a combination of HPLC and massselective detector (MSD) TOF. The liquid chromatography (LC) system will separate the different pigments before the identification/quantification takes place. The high mass accuracy of the LC/MSD TOF allows the user to confirm the identity as well as the quantity of each compound analyzed. Due to the better precision and accuracy of the mass measurement, LC/MSD TOF appears to represent a very precise analytical method, especially for the identification of unknown pigments.

\section{Conclusion}

As indicated in this chapter, several analytical methods are used in the identification of pigments in tattoo products, paint, and paintings. The choice of method will depend on the purpose, e.g. pigment identification/quantification or identification only. As mentioned in the introduction, the chemical identification of pigments in tattoo products is important to establish a pig- ment-related safety profile, which has been the main focus of this chapter. When characterizing pigment degradation products and impurities, quantification methods will be necessary. For the routine laboratory, several analytical methods seem to be possible.

For the world of art, where the identification of pigments is a very important issue, the scientific literature points out FTIR to be the best choice. Forensic laboratories also use this method as standard option. The sample preparation is $\mathrm{mi}-$ nor, as the tattoo ink can be placed directly on the IR transparent window and evaporated, or the pigments can be filtrated and pressed in the $\mathrm{KBr}$ disc. As reference spectral data are necessary in the identification procedure, it is notable that several FTIR pigment reference databases are available (http://www.ir-spectra.com/2012/ indexes/index_p.htm).

HPLC has been used by several scientists and routine laboratories when separation of pigments is necessary. For example, the identification of the 35 banned pigments requires proper separation before final identification. The identification is based on comparison of retention times and spectral data from reference samples. Raman spectroscopy has been performed on paintings and tattoo ink as well. In the analysis of tattoo ink, the sample preparation seems to be rather comprehensive to avoid interfering fluorescence signals from $\mathrm{TiO}_{2}$. It should be mentioned that several Raman pigment reference databases exist [6]. All of the above-mentioned methods are suitable for routine laboratories. Once again, it should be noted that access to relevant pigment reference samples as well as spectral databases is crucial.

For the research laboratories with access to more advanced equipment, LC/MSD TOF, direct analysis in real time TOF MS, or matrix-assisted laser desorption ionization TOF with a DEP seem to be the state-of-the-art methods. Access to research laboratories is important in situations in which the routine methods are insufficient. 


\section{References}

1 Baeumler W, Vasold R, Lundsgaard J, Talberg HJ: Chemicals used in tattooing and permanent make up products; in Papameletiou D, Schwela D, Zenie A, Baeumler W (eds): Workshop on the technical/scientific and regulatory issues on the safety of tattoos, body piercing and related practices. Ispra, European Commission, 2003, pp 21-48.

-2 De Cuyper C, D’hollander D: Materials Used in Body Art; in De Cuyper C, Pérez-Cotapos S ML (eds): Dermatologic Complications with Body Art. Dordrecht, Springer-Verlag Berlin/Heidelberg, 2010, pp 13-28.

3 Prior G: Tattoo Inks: Analysis, Pigments, Legislation. Berlin, epubli, 2014.

4 Poon KWC, Dadour IR, McKibley J: In situ chemical analysis of modern organic tattooing inks and pigments by micro-Raman spectroscopy. J Raman Spectrosc 2008;39:1227-1237.

5 Council of Europe: Resolution ResAP(2008)1, on Requirements and criteria for the safety of tattoos and permanent make-up.

-6 Engel E, Vasold R, Bäumler W: Tätowierungspigmente im Focus der Forschung. Nachr Chem 2007;55:847851.

7 Vasold R, Naarmann N, Ulrich H, Fisher D, König B, Landthaler M, Bäumler W: tattoo pigments are cleaved by laser light- the chemical analysis in vitro provide evidence for hazardous compounds. Photochem Photobiol 2004;80: 185-190.

8 Hauri U: Inks for tattoos and PMU (permanent make-up)/organic pigments, preservatives and impurities such as primary aromatic amines and nitrosamines. State Laboratory of the Canton Basel City. 2011. Available from: www.kantonslabor-bs.ch/files/ berichte/6729_111012_JB_Tattoo_ PMU_2011_EN.pdf.
9 Bäumler W, Eibler ET, Hohenleutner U, Sens B, Sauer J, Landthaler M: Q-Switch laser and tattoo pigments: first results of the chemical and photophysical analysis of 41 compounds. Lasers Surg Med 2000;26:13-21.

10 Timko AL, Miller CH, Johnson FB: In vitro quantitative chemical analysis of tattoo pigments. Arch Dermatol 2001; 137:143-147.

11 Danish Environmental Protection Agency: Chemical Substances in Tattoo Ink. Survey of chemical substances in consumer products (Kortlægning af kemiske stoffer i forbrugerprodukter) No. 116, 2012.

12 Vandenabeele P, Verpoort F, Moens L: Non-destructive analysis of paintings using Fourier transform Raman spectroscopy with fibre optics. J Raman Spectrosc 2001;32:263-269.

13 Vila A, Garcia FJ: Analysis of the chemical composition of red pigments and inks for the characterization and differentiation of contemporary prints. Anal Lett 2012;45:1274-1285.

14 Schaening A, Schreiner M, JembrihSimburger: Identification and classification of synthetic organic pigments of a collection of the 19th and 20th century by FTIR; in Picollo M (ed): The Sixth Infrared and Raman Users Group Conference (IRUG6), 29.3.-1.4., Florence, Italy, 2004, pp 302-305.

15 Schäning A, Varmuza K, Schreiner M: Pigment classification of synthetic organic pigments by multivariate data analysis of FTIR spectra. e-PS 2009;6: 75-80.

16 Learner T: The use of a diamond cell for the FTIR characterization of paints and varnishes available to twentieth century artists. Postprints: IRUG2 Meeting, pp. 7-20. Available at http://www.getty.edu/ conservation/our_projects/science/ modpaints/1Learner.pdf.

17 FBI Laboratory Chemistry Unit: FTIR Analysis of Paints, Tapes, and Polymers. FBI Laboratory Chemistry Unit SOP Manual PPSU 200-0.doc, 2006, pp 1-12.
18 Bhakti R, Petigara B: Separation and identification of pigments found in permanent cosmetic/tattoo inks using reversed-phase high performance liquid chromatography and photodiode array detection. Abstracts of the 230th ACS National Meeting, August 28-September 1, Washington, DC, 2005.

19 Cui Y, Spann AP, Couch LH, Gopee NV, Evans FE, Churchwell MI, Williams LD, Doerge DR, Howard PC: Photodecomposition of Pigment Yellow 74, a pigment used in tattoo inks. Photochem Photobiol 2004;80:175-184.

20 Engel E, Santarelli F, et al: Establishment of an extraction method for the recovery of tattoo pigments from human skin using HPLC diode array detector technology. Anal Chem 2006;15:78: 6440-6470.

21 Deutsches Institut für Normung: Textilien-Nachweis von Dispersionsfarbstoffen. DIN 54231, 2005. Available at: http://www.din.de/cmd\%3Bjsessionid=J D9UB2Z2AKIJVC2RHGRWHYZX.1?wo rkflowname $=$ infoInstantdownload $\&$ doc name $=9642857 \&$ contextid $=$ din $\&$ service refname $=$ dino\&ixos $=$ toc.

22 Analysis of organic pigments using a direct exposure probe on JMS-T100GC 'AccuTOF GC' Jeol MS Data Sheet no 085, 2006.

23 Djelal H, Cornée C, Tartivel R, Lavastre O, Abdeltif A: The use of HPTLC and Direct Analysis in Real Time-Of-Flight Mass Spectrometry (DART-TOF-MS) for rapid analysis of degradation by oxidation and sonication of an azo dye. Arabian J Chem 2013. http://dx.doi. org/10.1016/j.arabjc.2013.06.003

24 Fang Y, Li P, Zumwalt M: Determination of EU-Banned Disperse Dyes by LC/MSD TOF. Agilent Technologies, 2005. Available at: http://www.chem.agilent.com/ Library/applications/5989-3859EN.pdf.

Ms. Sci. Chem. Ole Olsen

Medico Chemical Lab ApS

Skelstedet 5

DK-2950 Vedbaek (Denmark)

E-Mail OO@medico.dk 